\title{
A refractory heterogeneous Cryopyrin-Associated Periodic Syndrome (CAPS) phenotype related to V198M mutation responds to canakinumab - a case report
}

\author{
Sandra Hansmann, Christiane Zimmer, Monika Moll, Nikolaus Rieber, Jasmin B Kuemmerle-Deschner* \\ From 18th Pediatric Rheumatology European Society (PReS) Congress \\ Bruges, Belgium. 14-18 September 2011
}

\section{Background}

V198M mutation has been identified in more than one CAPS phenotype and is difficult to treat.

\section{Aim}

To report effects of prolonged selective IL- $1 \beta$ inhibition with canakinumab in a CAPS phenotype related to V198M mutation.

\section{Methods}

A 9-year old boy, diagnosed with symptomatic MWS and evidence of a $V 198 M$ mutation (father also $V 198 M)$, only partially responsive to anakinra, participated in a phase III study of canakinumab in CAPS patients $(\mathrm{N}=166)$. Study treatment was 8 -weekly administration of canakinumab by s.c. injection, $150 \mathrm{mg}$ to adults and $2 \mathrm{mg} / \mathrm{kg}$ to patients $\leq 40 \mathrm{~kg}$ for up to 2 years. Administration frequency and dose (maximum $600 \mathrm{mg}$ or $8 \mathrm{mg} / \mathrm{kg}[\leq 40 \mathrm{~kg}]$ ) were increased in case of residual symptoms.

\section{Results}

After insufficient response to per-protocol canakinumab dose, the patient received high dose canakinu$\mathrm{mab}(10 \mathrm{mg} / \mathrm{kg}$, i.v. $)$ every 4 weeks from Day 20 onwards to $>440$ days. Disease activity improved from severe to moderate at Day 48 and remained stable including normal CRP and SAA values $(<10$ $\mathrm{mg} / \mathrm{L}$ ). Temporary mild adverse events (AE) were not suspected to be treatment-related (upper respiratory viral infection, gastroenteritis, and rhinitis). Night sweats, dry lips and skin persisted until last assessments. No serious AEs/infections were reported in this patient.

\section{Conclusion}

Based on our experience in this patient and a review of literature on efficacy of IL-1 inhibition in CAPS patients with V198M mutation, high dose canakinumab i.v* with increased dosing frequency yields symptomatic relief without evidence of increased AE. Confirmation of our observation in more patients with similar genetic and clinical presentation is needed.

*The posology used for this case is not in line with the approved posology of canakinumab

Published: 14 September 2011

doi:10.1186/1546-0096-9-S1-P9

Cite this article as: Hansmann et al:: A refractory heterogeneous Cryopyrin-Associated Periodic Syndrome (CAPS) phenotype related to V198M mutation responds to canakinumab - a case report. Pediatric Rheumatology 2011 9(Suppl 1):P9. 